\title{
Sensitivity of simulated yield of dryland wheat to uncertainty in estimated plant-available water capacity
}

\author{
$\underline{\text { Chao Chen }}^{\mathrm{a}}$, Renye Wu ${ }^{\mathrm{b}, \mathrm{a}}$, Andrew Fletcher ${ }^{\mathrm{a}}$, Roger Lawes ${ }^{\mathrm{a}}$, Yvette Oliver ${ }^{\mathrm{a}}$ \\ ${ }^{a}$ CSIRO Agriculture Flagship, Private Bag 5, PO Wembley WA 6913, Australia \\ ${ }^{b}$ College of crop sciences, Fujian Agriculture and Forestry University, Fuzhou, 350002, China \\ Email:chao.chen@,csiro.au
}

\begin{abstract}
Water availability, which is determined by rainfall and also by the plant available water holding capacity (PAWC) of soils, is a key determinant of crop yield in the Mediterranean farming systems of the Western Australian wheatbelt. While unlike rainfall data, soil parameters (DUL: drained upper limit, CLL: crop lower limit and RD: rooting depth) affecting PAWC at a field scale rarely exist and the values required by crop simulation models (e.g. APSIM) have to be inferred from nearby soil surveys or local knowledge. The estimation of these soil parameters unavoidably creates uncertainty in PAWC, which results in uncertainty in simulated crop yield. There may also be interactive effects on simulated crop yield between biased estimate of PAWC, rainfall and nitrogen $(\mathrm{N})$ supply. However, the sensitivity and uncertainty of simulated crop yield to errors in estimated PAWC under variable rainfall and fertilizer applications have not been well researched. This study performed a sensitivity analysis, using the APSIM model, to quantify the influence of the uncertainties in PAWC on estimation of wheat yield in a rain-fed Mediterranean type environment with one typical soil and four $\mathrm{N}$ treatments. Simulation results showed that, for any given amount of N, the changes in the three parameters (DUL, CLL and RD) that resulted in the same changes in PAWC would result in the same changes in wheat yield, i.e. PAWC is the key factor to affect simulated yield. When PAWC was biasedly estimated by a small amount $(< \pm 20 \mathrm{~mm})$, the impact on simulated wheat yield was marginal $(0-200 \mathrm{~kg} / \mathrm{ha})$ under all $\mathrm{N}$ treatments. However, when changes in PAWC were larger $(> \pm 20 \mathrm{~mm})$, its biased estimation had assymetric effects on simulated rainfed wheat yields: on average the underestimation of PAWC would cause greater deviation in simulated wheat yield compared to its overestimation regardless of $\mathrm{N}$ treatment. For example, when PAWC was underestimated by $40 \mathrm{~mm}$, wheat yield was reduced by $80,170,320$ and $440 \mathrm{~kg} / \mathrm{ha}$ under zero, low, medium and high $\mathrm{N}$ treatments, respectively. In contrast when PAWC was increased by 40 $\mathrm{mm}$, simulated yield increased by $30,70,140$ and $240 \mathrm{~kg} /$ ha under the four $\mathrm{N}$ treatments, respectively. There was an interactive effect on simulated wheat yield between PAWC, $\mathrm{N}$ supply and seasonal rainfall, i.e. the simulated yield bias was larger when PAWC was estimated with bias in situations with more rainfall and more fertilizer N. Ideally simulations should be based on accurately measured soil water parameters. However, in the absence of measured data an overestimation of the true PAWC will result in less error in simulated yields than and underestimation, irrespective of rainfall condition and soil $\mathrm{N}$ supply.
\end{abstract}

Keywords: $\quad$ APSIM, PAWC, wheat, soil parameters 


\section{INTRODUCTION}

Dynamic crop models have been widely used for agricultural analysis such as crop monitoring, yield prediction or agricultural resource management (Varella, Guérif \& Buis 2009). Simulation of the behaviour of crops involves a number of processes such as photosynthesis, dry matter partitioning, crop development and transpiration (Aggarwal 1995). These processes are affected by soil and weather factors, crop type and management, which are required as inputs or parameters to run the models. It is generally easy to obtain historic climate information for specific locations in Australia, but the knowledge on soil properties and therefore model requiring soil parameters is scarcely available at an appropriate scale. When crop models like APSIM that require soil hydrological inputs of drained upper limit (DUL), crop lower limit (CLL) and rooting depth (RD) are used to simulate crops at regional or larger spatial scales, it is often difficult to precisely characterise these critical attributes of the soil, due to the inherent variability in natural processes, costly monitoring, or imperfections in data measurements (Wang et al. 2005; Wu et al. 2010). As a result these parameters are often estimated based on nearby soil surveys or local knowledge.

In the Mediterranean-type climatic wheatbelt of Western Australia (WA), as in many of the world's dryland agricultural regions, crop production depends heavily on rainfall and plant-available water capacity (PAWC), the latter of which is the difference between DUL and CLL over a crop's RD (Dalgliesh \& Foale 1998). The uncertainty estimates of DUL, CLL or RD inevitably results into uncertainties in PAWC, which is expected to lead to biased estimates of crop yield in water-limited environments (Aggarwal 1995; Tremblay \& Wallach 2004; Makowski et al. 2006). However, to the best of our knowledge, only a few studies have been conducted to quantify the uncertainty in simulated yield considering uncertainties in PAWC. Our previous study (Wu et al., Unpublished) examined the effect of imprecise estimates of PAWC on rainfed wheat using the APSIM model, and found that a very similar bias in simulated dryland wheat potential yield would be caused if biased estimation of DUL, CLL and RD caused the same uncertainty in PAWC, measurement errors that underestimated or overestimated PAWC by up to $20 \mathrm{~mm}$ only had a marginal impact on simulated potential yield. However, it is still unclear what the response patterns of uncertainties in PAWC to the three soil hydrological parameters and biased estimate of yield to the uncertainties in PAWC might look like under different levels of fertilizer.

The objective of this study was to re-quantify the bias in simulated wheat yield due to the uncertainties of PAWC caused by inputs of DUL, CLL and RD in the environment characterized by variable rainfall and different nitrogen。

\section{MATERIALS AND METHODS}

\subsection{APSIM overview}

APSIM, the Agricultural Production Systems Simulator is a process-based dynamic crop model that combines biophysical and management modules within a central engine to simulate diverse cropping systems (Holzworth et al. 2014). The model is driven by daily climate data and is able to simulate growth, development and yield of crops and their interactions with soil. In order to model wheat-based cropping systems, four modules were linked wheat crop (Wheat), soil water (SOILWAT), soil nitrogen (SOILN) and soil residue (RESIDUE).

In APSIM, potential wheat production is calculated using stage-related radiation-use efficiency which is then limited to actual above-ground biomass production on a daily basis by soil water and $\mathrm{N}$ availability (Keating et al. 2003). The APSIM-Wheat module that simulates wheat growth processes seeks information regarding water and $\mathrm{N}$ availability directly from SOILWAT and SOILN modules on a daily basis. The SOILWAT module simulates the various vertical water movements in a layered soil system using a multi-layer cascading approach. Soil water characteristics are specified in terms of air dry, lower limit at 15 bar, DUL and saturated volumetric water contents in the crop rooting zone. The CLL parameter that describes the plant's ability to extract water from the soil is also needed to specify the absolute water supply of the crop. PAWC is subsequently determined by the difference between DUL and CLL over RD. Mineralisation and immobilisation of soil N are simulated in the APSIM-SOILN module.

\subsection{Study sites and climate data}

The sensitivity analysis was conducted using historical long-term daily climate data (1951-2015) at Wongan Hills in the medium rainfall zone in the wheatbelt of WA. Average annual rainfall was about $490 \mathrm{~mm}$, with $370 \mathrm{~mm}$ falling during wheat growing season (May-October). For all simulations, the soil water profile was 
reset to CLL on 1 January each year and soil $\mathrm{N}$ was reset to $50 \mathrm{~kg} \mathrm{~N}$ at sowing time. The wheat cultivar Mace was used throughout the simulations. Wheat was sown when $20 \mathrm{~mm}$ of rainfall was accumulated over a fiveday period between May 1 and June 30 .

\subsection{Modelling the effects of the uncertainties in PAWC on rainfed wheat yield under different levels of fertilizer $\mathbf{N}$}

Loamy sand, occupying the largest percent of the area in the wheatbelt of WA, was selected to evaluate the effects of the uncertainties in PAWC resulted from biased estimates in DUL, CLL and RD on rainfed wheat yield. The original characteristics of the soil were derived from field measurements as described by Asseng et al. (1998), with a PAWC value of $140 \mathrm{~mm}$. To quantify the effects of uncertain inputs in PAWC on simulated wheat, a series of changes were made to PAWC by changing DUL, CLL and RD, respectively, relative to their standard values: $-60 \mathrm{~mm},-40 \mathrm{~mm},-20 \mathrm{~mm}, 0 \mathrm{~mm}, 20 \mathrm{~mm}, 40 \mathrm{~mm}$ and $60 \mathrm{~mm}$ (when one parameter was changed, the others were kept unchanged). Simulations were conducted with four levels of fertilizer N, $0 \mathrm{~kg}$ $\mathrm{N} / \mathrm{ha}, 30 \mathrm{~kg} \mathrm{~N} / \mathrm{ha}$ (low), $60 \mathrm{~kg} \mathrm{~N} / \mathrm{ha}$ (medium) and $90 \mathrm{~kg} \mathrm{~N} / \mathrm{ha}$ (high) to allow sufficient exploration of the effect of biased estimate in PAWC under limitation by nitrogen.

\section{RESULTS AND DISCUSSIONS}

\subsection{Re-quantification of the relationship in the uncertainties in PAWC and yield estimation}

The response of average change in simulated wheat yield under different levels of fertilizer $\mathrm{N}$ with the change in PAWC as affected by DUL, CLL or RD are shown in Fig. 1. Under all levels of fertilizer N supply, the overestimation of PAWC caused the overestimation of wheat yield, and vice versa. But the amplitude of the change in wheat was different depending on the $\mathrm{N}$ availability. The amplitude of the biased estimate of yield was larger under high $\mathrm{N}$ treatment. Under a specific level of $\mathrm{N}$ fertilizer supply, the average changes in yield showed similar response patterns to the changes in PAWC, no matter which parameter (DUL, CLL or RD) caused the error in PAWC (Fig. 1 a-d). These results indicate that, on average, the three hydrological parameters, i.e. DUL, CLL and DUL would have very similar effects on the change in simulated wheat yield for any levels of fertilizer $\mathrm{N}$ supply, if the biased estimates of them caused the same amount of variation of PAWC. It is therefore recommended that attention should be given to more accurately estimating a soil's water holding capacity, rather than a specified soil parameter. Compared to the effects of overestimation of PAWC, the underestimation of PAWC had more severe effects on estimates of wheat yield (Fig. 1 a-d). For example an overestimation of $40 \mathrm{~mm}$ of PAWC would result into an overestimation of $30,70,140$ and $240 \mathrm{~kg} / \mathrm{ha}$ under
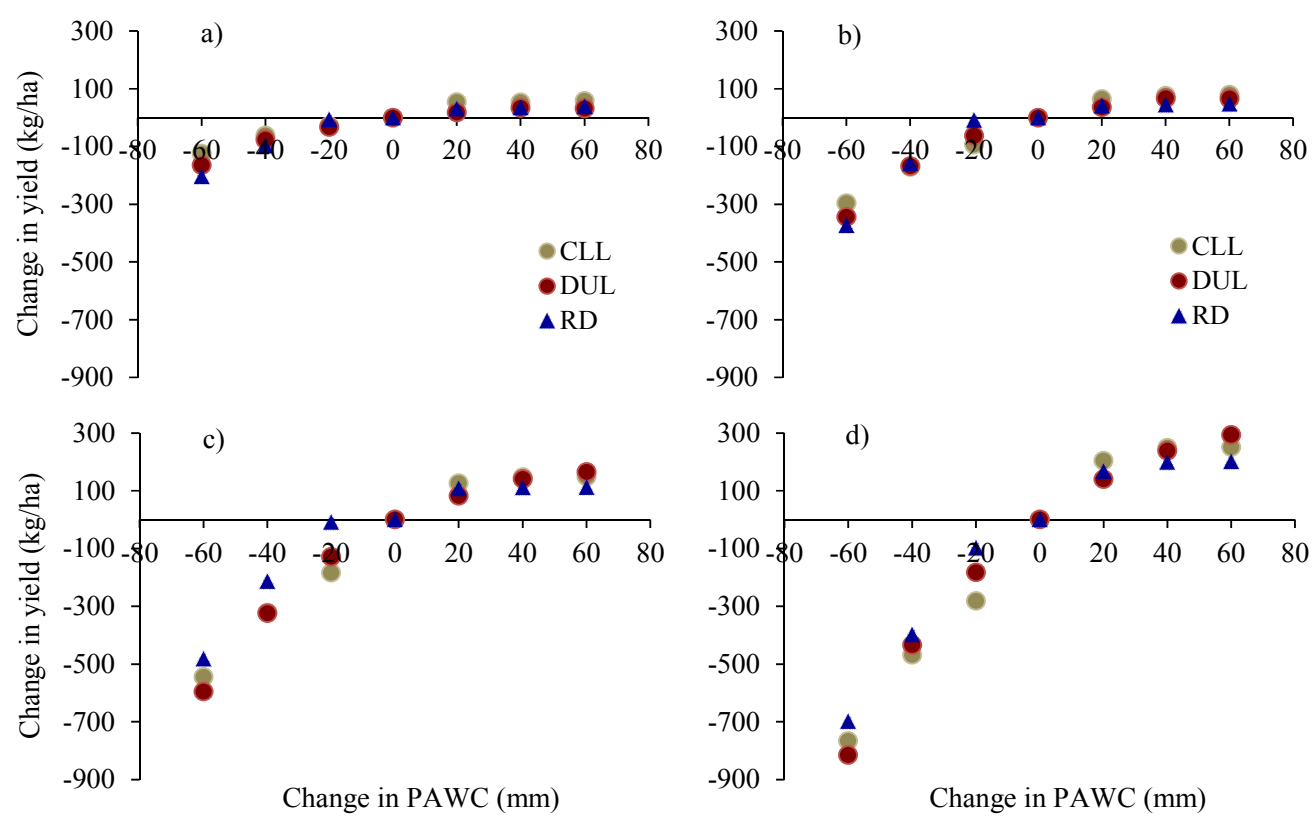

Figure 1. Relationship between the change in simulated average rainfed wheat yield and change in plant available water capacity (PAWC) as affected by the change in drained upper limit (DUL), crop lower limit (CLL) and root depth (RD) for zero N (a), low N (b), medium N (c) and high N (d)) treatments at Wongan Hills, WA. 
zero, 30, 60 and $90 \mathrm{~kg} \mathrm{~N} / \mathrm{ha}$ applications, respectively; while the same amount of underestimation of PAWC would cause the underestimation of wheat yield for $80,170,320$ and $440 \mathrm{~kg} / \mathrm{ha}$ under the four $\mathrm{N}$ treatments, respectively (Fig. 1 a-d). It is important to measure all three soil hydrological parameters as accurately as possible to reduce the uncertainties in simulate yield, but overestimation for PAWC rather than underestimation should be considered when soil parameters have to be estimated, especially when $\mathrm{N}$ availability was high.

\subsection{Effects of uncertainty in PAWC on rainfed wheat yield under variable rainfall and different $\mathbf{N}$ availability}

Under all levels of fertilizer $\mathrm{N}$, the changes in simulated wheat yield responded similarly to DUL, CLL or RD if they caused same amount of bias of PAWC. Here the overestimation and underestimation of PAWC caused by DUL was used to analyse the response of biased estimates of wheat yield to the uncertainties in PAWC under variable rainfall and different rates of fertiliser $\mathrm{N}$.

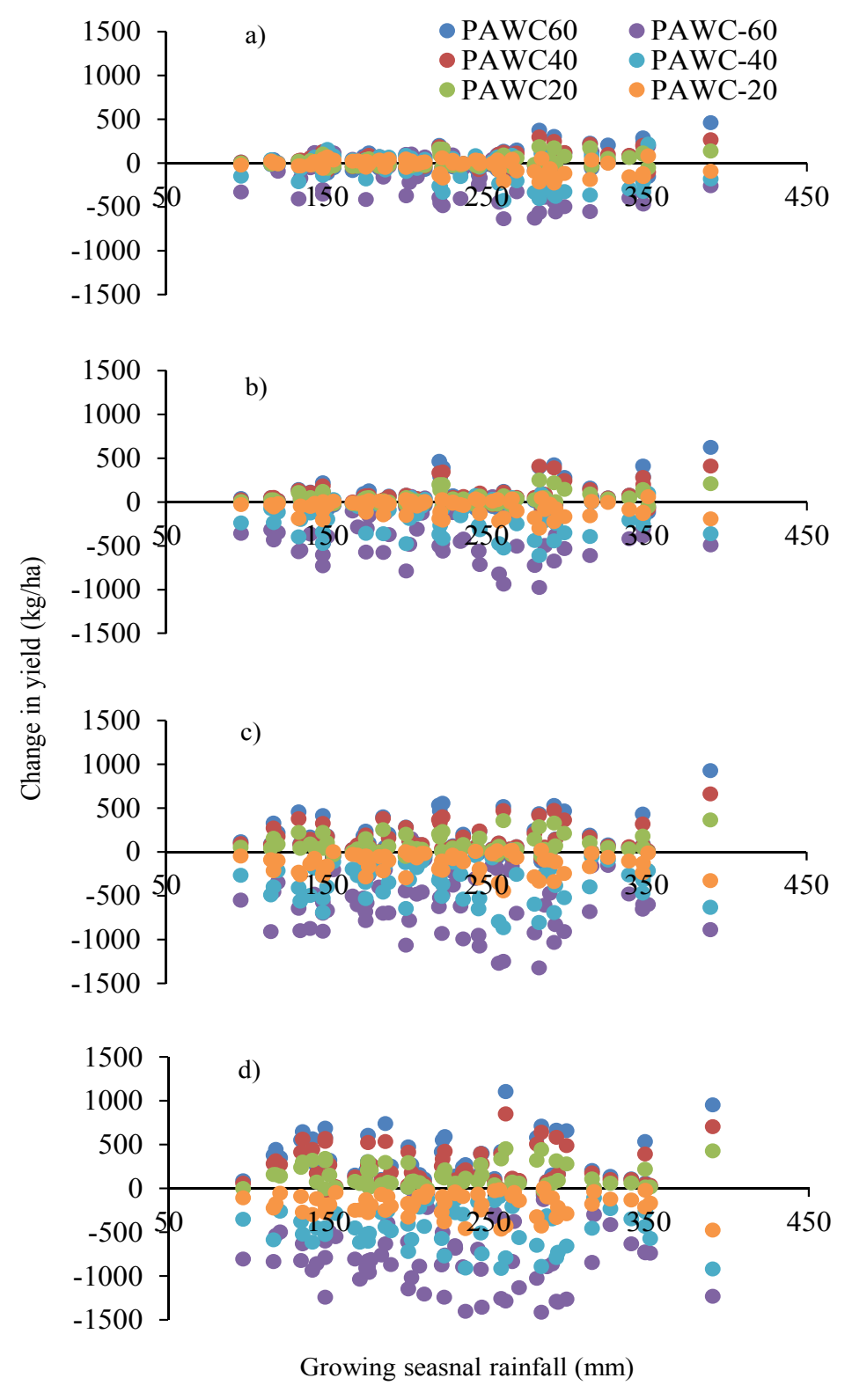

Figure 2. The response of the change in simulated rainfed wheat yield as affected by the increase or decrease in PAWC to wheat growing season rainfall under zero $\mathrm{N}(\mathrm{a})$, low $\mathrm{N}(\mathrm{b})$, medium $\mathrm{N}(\mathrm{c})$ and high $\mathrm{N}(\mathrm{d})$ treatments, respectively; Note: PAWC20, PAWC40 and PAWC60 mean that PAWC was increased by 20, 40 and $60 \mathrm{~mm}$, respectively by changing DUL; PAWC-20, PAWC-40 and PAWC-60 mean that PAWC was decreased by 20, 40 and $60 \mathrm{~mm}$, respectively by changing DUL. 
The response of overestimating or underestimating wheat yield caused by overestimating or underestimating PAWC under different levels of fertilizer N supply is shown in Fig. 2. Rainfall had a clear effect on the bias of the estimated wheat yield as affected by the uncertainties in PAWC under all levels of fertilizer N. When PAWC was overestimated, the amplitude of the overestimation of wheat yield was enlarged with the growingseason rainfall, and the trend was more obvious under high $\mathrm{N}$ treatment. Under this condition, the overestimation of yield is larger because the overestimated PAWC allows the soil to hold more rainfall when it is available, which, together with $\mathrm{N}$, causes the higher yield estimation.

Once growing season rainfall exceeded $300 \mathrm{~mm}$, the impact of PAWC on wheat yield estimate declined, because $300 \mathrm{~mm}$ of rainfall roughly meets the water demand of a wheat crop in these regions (Oliver et al. 2009). When PAWC was underestimated, the magnitude of the underestimation of wheat yield increased with growing-season rainfall, and this increased rate with rainfall was faster for larger PAWC underestimation (Fig. $2 b)$. The magnitude of biased wheat yields due to the uncertainties of PAWC was larger in high rainfall season with high fertilizer N supply, compared with a low rainfall season with low $\mathrm{N}$. This is because an overestimated PAWC makes the soil have the ability to store more water which leads to larger water availability causing higher yield estimation under high $\mathrm{N}$ availability. Therefore if a season is wet with high $\mathrm{N}$ availability, the importance of estimating PAWC precisely is increased.

In conclusion, if a biased estimation of the parameters of DUL, CLL or RD resulted in the same error in estimated PAWC, then the error in simulated rainfed wheat yield would be similar, regardless of $\mathrm{N}$ status. To obtain acceptable rainfed yield simulations in the wheatbelt of WA, bias in the estimations of PAWC need to be less than $20 \mathrm{~mm}$. The underestimation of PAWC would incur more severe deviation of rainfed yield estimates than the same amount of its overestimation. We conclude that, to reduce the uncertainties in simulated yield, it is important to measure all three soil hydrological parameters as accurately as possible, but overestimation of PAWC rather than underestimation should be made when these parameters have to be estimated.

\section{ACKNOWLEDGMENTS}

This research was supported by the Grains Research and Development Corporation project BWD00025 'National Paddock Survey'.

\section{REFERENCES}

Aggarwal, P. (1995). Uncertainties in crop, soil and weather inputs used in growth models: Implications for simulated outputs and their applications. Agricultural Systems, 48, 361-384.

Asseng, S., Keating, B.A., Fillery, I.R.P., Gregory, P.J., Bowden, J.W., Turner, N.C., Palta, J.A. and Abrecht, D.G. (1998). Performance of the APSIM-wheat model in Western Australia. Field Crops Research, 57 , 163-179.

Dalgliesh, N. and Foale, M.A. (1998). Soil matters: monitoring soil water and nutrients in dryland farming. Agricultural Production Systems Research Unit Toowoomba, Qld.

Holzworth, D.P., Huth, N.I., Devoil, P.G., Zurcher, E.J., Herrmann, N.I., McLean, G., Chenu, K., van Oosterom, E.J., Snow, V., Murphy, C., Moore, A.D., Brown, H., Whish, J.P.M., Verrall, S., Fainges, J., Bell, L.W., Peake, A.S., Poulton, P.L., Hochman, Z., Thorburn, P.J., Gaydon, D.S., Dalgliesh, N.P., Rodriguez, D., Cox, H., Chapman, S., Doherty, A., Teixeira, E., Sharp, J., Cichota, R., Vogeler, I., Li, F.Y., Wang, E.L., Hammer, G.L., Robertson, M.J., Dimes, J.P., Whitbread, A.M., Hunt, J., van Rees, H., McClelland, T., Carberry, P.S., Hargreaves, J.N.G., MacLeod, N., McDonald, C., Harsdorf, J., Wedgwood, S. and Keating, B.A. (2014). APSIM - Evolution towards a new generation of agricultural systems simulation. Environmental Modelling and Software, 62, 327-350.

Keating, B.A., Carberry, P.S., Hammer, G.L., Probert, M.E., Robertson, M.J., Holzworth, D., Huth, N.I., Hargreaves, J.N.G., Meinke, H., Hochman, Z., McLean, G., Verburg, K., Snow, V., Dimes, J.P., Silburn, M., Wang, E., Brown, S., Bristow, K.L., Asseng, S., Chapman, S., McCown, R.L., Freebairn, D.M. and Smith, C.J. (2003). An overview of APSIM, a model designed for farming systems simulation. European Journal of Agronomy, 18, 267-288.

Makowski, D., Naud, C., Jeuffroy, M.-H., Barbottin, A. and Monod, H. (2006). Global sensitivity analysis for calculating the contribution of genetic parameters to the variance of crop model prediction. Reliability Engineering and System Safety, 91, 1142-1147. 
Chen et al., Sensitivity of simulated yield of wheat to uncertainty in plant-available water capacity

Oliver, Y., Robertson, M., Stone, P. and Whitbread, A. (2009). Improving estimates of water-limited yield of wheat by accounting for soil type and within-season rainfall. Crop and Pasture Science, 60, 1137-1146.

Tremblay, M. and Wallach, D. (2004). Comparison of parameter estimation methods for crop models. Agronomie, 24, 351-365.

Varella, H., Guérif, M. and Buis, S. (2009). Estimation of Soil Properties Using Observations and the Crop Model STICS. Interest of Global Sensitivity Analysis and Impact on the Prediction of AgroEnvironmental Variables. INTECH Open Access Publisher.

Wang, X., He, X., Williams, J., Izaurralde, R. and Atwood, J. (2005). Sensitivity and uncertainty analyses of crop yields and soil organic carbon simulated with EPIC. Transactions-American Society of Agricultural Engineers, 48, 1041.

Wu, W., Chen, J.-1., Liu, H.-b., y Garcia, A.G. and Hoogenboom, G. (2010). Parameterizing soil and weather inputs for crop simulation models using the VEMAP database. Agriculture, Ecosystems and Environment, $135,111-118$. 